\title{
Review of: "Exploring the effect of image enhancement techniques on COVID-19 detection using chest X-ray images"
}

Mizuho Nishio

Potential competing interests: The author(s) declared that no potential competing interests exist.

\section{Affiliation}

Department of Radiology, Kobe University Graduate School of Medicine, 7-5-2 Kusunoki-cho, Chuo-ku, Kobe, 650-0017, Japan. nishiomizuho@gmail.com.

Rahman et al. investigated the automatic classification of chest X-ray (CXR) images into COVID-19 pneumonia, non-COVID-19 pneumonia, and normal CXRs using Convolutional Neural Network (CNN) models [1]. The major contributions of this study were as follows: (i) the study evaluated the effect of lung segmentation and image enhancement techniques on the classification and (ii) the authors constructed a large dataset of COVID-19 pneumonia, non-COVID-19 pneumonia, and normal CXR images. The authors' results show that Gamma correction was useful for improving the performance of CNNs. Because I am interested in image preprocessing for CNNs, I find this paper to be useful.

Major points

\section{1}

The authors show that their modified U-net could perform reliable lung segmentation for their dataset. However, their dataset includes many normal CXR images (nearly 50\% of CXRs in the test set are normal). Therefore, their modified U-net may not perform well in CXR images of COVID-19 pneumonia and nonCOVID-19 pneumonia. As shown in one paper [2], the performance of their modified U-net should be evaluated for CXR images with severe abnormal findings.

2

The classification's performance was slightly better on plain CXR images than on segmented lung CXR images; this might have been caused by the performance degradation of the authors' modified U-net for CXR images with severe abnormal findings. 
It seems that the authors intend to disclose the dataset they used in this study. I hope that their dataset will be disclosed immediately, to promote the automatic classification of CXR images.

4

According to their paper, their definitions of true positive (TP), true negative (TN), false positive (FP), and false negative (FN) are as follows:

- TP: the number of COVID-19 CXR images that were identified as COVID-19 CXRs

- TN: the number of normal and non-COVID lung opacity CXRs that were identified as normal and nonCOVID CXRS

- FP: the number of normal and non-COVID CXRs that were incorrectly identified as COVID-19 CXRs

- FN: the number of COVID-19 CXRs that were incorrectly identified as normal and non-COVID CXRs

In my opinion, these definitions may be inadequate. For example, if a normal CXR image was identified as non-COVID, how did the authors handle this result? Such a result may have been treated as a TN in this study. However, this result would be regarded as a misdiagnosis from a clinical viewpoint. The results of the performance metrics of this study should therefore be handled carefully.

Minor points

5

EfficientNet and Vision Transformer are both state-of-the-art and are available as CNNs with pretrained models $[3,4]$. I am interested in how these models would perform on the authors' dataset.

\section{Acknowledgments}

For writing this commentary, the author was partly supported by JSPS KAKENHI (grant number: 19H03599 and JP19K17232).

\section{References}

1. Rahman T, Khandakar A, Qiblawey Y, Tahir A, Kiranyaz S, Abul Kashem S Bin, et al. Exploring the effect of image enhancement techniques on COVID-19 detection using chest X-ray images. Comput Biol Med. 
2021;132: 104319. doi:10.1016/J.COMPBIOMED.2021.104319

2. Nishio M, Fujimoto K, Togashi K. Lung segmentation on chest X-ray images in patients with severe abnormal findings using deep learning. Int J Imaging Syst Technol. 2021;31: 1002-1008. doi:10.1002/IMA.22528

3. Tan M, Le Q. EfficientNet: Rethinking Model Scaling for Convolutional Neural Networks. Proceedings of the 36th International Conference on Machine Learning. 2019. pp. 6105-6114. Available: https://proceedings.mlr.press/v97/tan19a.html

4. Dosovitskiy A, Beyer L, Kolesnikov A, Weissenborn D, Zhai X, Unterthiner T, et al. An Image is Worth 16x16 Words: Transformers for Image Recognition at Scale. Proceedings of ICLR 2021. 2021. Available: https://github.com/ 